\title{
ON THE GROWTH RATE OF THE CORRELATION FUNCTION OF FAINT GALAXIES
}

\author{
DAVID VALLS-GABAUD ${ }^{1}$ AND BOUD ROUKEMA ${ }^{2}$ \\ 1 Observatoire de Strasbourg, \\ 11 Rue de l'Université, 67000 Strasbourg, France. \\ ${ }^{2}$ Astronomy Centre, University of Sussex, Brighton BN1 9QH, \\ $U K$.
}

We constrain the growth rate of structure, as represented by the spatial two-point galaxy auto-correlation function, at redshifts where this has not yet been measured directly by combining recent measurements of the amplitude of the angular two-point galaxy auto-correlation, at magnitudes as faint as $V_{\text {median }} \leq 25$, with new observations of the redshift distribution of very faint galaxies. We show that $\xi$ for the overall galaxy population (at a fixed proper separation $r$ ) grows $(1+z)^{4 \pm 1}$ times as fast as clustering which is fixed in proper coordinates. Even extreme models where "blue" galaxies have a smaller, IRAS-like, correlation function do not reduce the growth rate below $(1+z)^{2.5 \pm 1}$ times the clustering fixed in proper coordinates (Roukema, B.F. and Valls-Gabaud, D. (1995) $A \mathscr{E} A$, submitted).

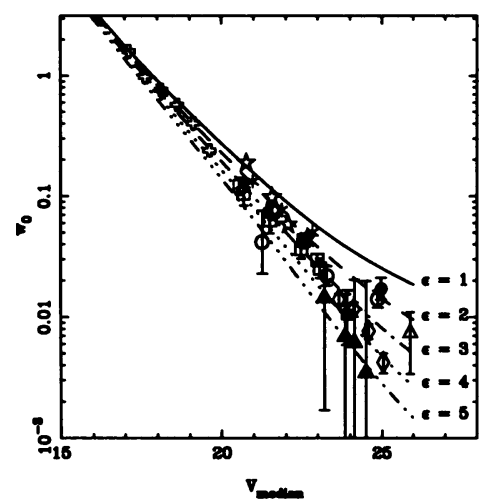

Figure 1. Amplitude of the galaxy two-point angular auto-correlation function $w_{0}=w\left(1^{\prime}\right)$ against median $V$ magnitude. 